\title{
Energy efficient chaotic whale optimization technique for data gathering in wireless sensor network
}

\author{
Sridhar R. ${ }^{1}$, N. Guruprasad ${ }^{2}$ \\ ${ }^{1}$ Department of Information Science and Engineering, Global Academy of Technology, India \\ ${ }^{2}$ Department of Computer Science and Engineering, Global Academy of Technology, India
}

\begin{tabular}{l}
\hline Article Info \\
\hline Article history: \\
Received Jun 28, 2019 \\
Revised Feb 5, 2020 \\
Accepted Feb 25, 2020 \\
\hline
\end{tabular}

Keywords:

Chaotic tent map

Chaotic whale metaheuristic

optimization

Data gathering

Residual energy

WSN

\begin{abstract}
A Wireless Sensor Network includes the distributed sensor nodes using limited energy, to monitor the physical environments and forward to the sink node. Energy is the major resource in WSN for increasing the network lifetime. Several works have been done in this field but the energy efficient data gathering is still not improved. In order to amend the data gathering with minimal energy consumption, an efficient technique called chaotic whale metaheuristic energy optimized data gathering (CWMEODG) is introduced. The mathematical model called Chaotic tent map is applied to the parameters used in the CWMEODG technique for finding the global optimum solution and fast convergence rate. Simulation of the proposed CWMEODG technique is performed with different parameters such as energy consumption, data packet delivery ratio, data packet loss ratio and delay with deference to dedicated quantity of sensor nodes and number of packets. The consequences discussion shows that the CWMEODG technique progresses the data gathering and network lifetime with minimum delay as well as packet loss than the state-of-the-art methods.
\end{abstract}

Copyright $@ 2020$ Institute of Advanced Engineering and Science. All rights reserved.

\section{Corresponding Author:}

Sridhar R.,

Department of Information Science and Engineering,

Global Academy of Technology,

Bengaluru, India.

Email: srimln77@gmail.com

\section{INTRODUCTION}

Now a day's advances in miniaturization yet reasonably efficient wireless communication equipment and improved small-scale energy supplies that have combined with reduced manufacturing costs to make a new technological vision called wireless sensor network. In WSN, a group of sensors are deployed arbitrarily for monitoring and collecting the physical conditions and arranging the collected data at sink node for further processing. The WSN is employed for several real-time applications such as military, monitoring environment, agriculture, home automation, smart transportation, health care and so on. In WSNs, various sensor nodes convey their collected information to a far-away base station via sink node.

Figure 1 illustrates the WSN architecture where the nodes are scattered in the network. In a typical WSN topology, one can distinguish between ordinary wireless sensor nodes and base stations named sinks. The sink is usually connected to a power supply and it is capable of performing more complex operations. Wireless sensor nodes have ability to transfer raw sensed data to the sink. Due to economic reasons, nodes are usually powered by small size batteries that in most application scenarios are even impossible to replace or recharge. Every node in WSN shares the information to another node through the radio waves. Data gathering in WSN is the process of accumulating the statistics from the sensor nodes and directs towards a sink periodically and guided towards the base station. 


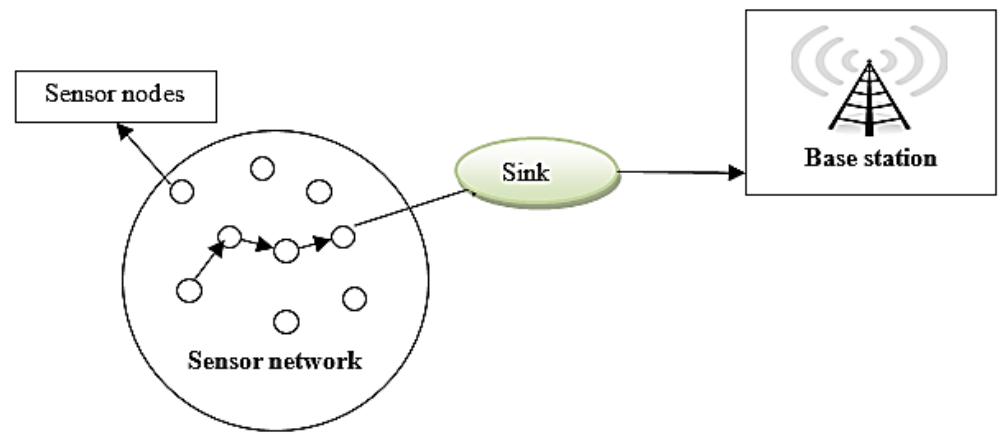

Figure 1. WSN architecture

The energy supplied to each sensor node are the essential challenges in WSNs due to high packet loss and delay. Therefore, efficient packet delivery ratio is also a significant problem in WSN. Another issue is the network may consist of a large number of rather different nodes in terms of sensors, computing power, and memory. Recent research proved that the optimization techniques provided as efficient energy conservation for data gathering in WSN.

A particle swarm optimization-based selection (PSOBS) Method was developed in [1] for amassing the statistics from the sensor nodes with the help of the mobile sink. Though the method reduces the energy utilization, the packet loss during the data gathering was not minimized. The hybrid ant colony optimization and particle swarm optimization (ACOPSO) Centered Energy Effective Clustering technique was introduced in [2] to strengthen the inter-cluster data amassment and enhance the network lifetime. The ACOPSO technique failed to improve the packet delivery ratio with minimum delay.

A maximum lifetime data aggregation tree scheduling (MLDATS) algorithm was introduced [3] to save the sensor nodes energy for attaining the improved network lifespan. But the algorithm failed to obtain efficient data aggregation in dynamic WSN. A Cluster-Ring Method was introduced [4] to improve the energy proficient gathering of data for extensive WSN. While changing the topology of network dynamically, the approach performed the data aggregation but failed to provide accurate results.

An Auction-Based Scheme was developed [5] for gathering the data from WSN with minimum energy consumption. The scheme failed to select the optimal sensor nodes for increasing the network lifetime. A Bayesian compressive sensing gathering of data method was introduced [6] for ensuring the data gathering and prolong the network lifetime. The approach failed to attain higher data packet delivery at the sink node. A Distributed Optimal Movement technique was developed [7] for collecting the data with minimal loss rate. But the energy efficient data gathering was not performed to increase the lifetime of network.

The rendezvous-based data congregation protocols were designed in [8] for delay inhibited data gathering in WSN. But the lifetime of network was not enriched since it failed to select the energy efficient nodes. An efficient structure-free data aggregation and delivery (ESDAD) technique was introduced [9] for improving the data delivery with lesser energy consumption. The reliability in the data aggregation process was not increased. A sparsity feedback-based compressive data gathering procedure was hosted [10] for balancing the vitality amid the sensor nodes. The procedure did not minimize the total energy consumption for further improving data gathering performance.

The major issues identified from the existing literature are higher packet loss rate, longer delay, lack of improving the network lifetime, failure to improve the data packet delivery and so on. In order to address such kind of issues, an efficient technique called chaotic whale metaheuristic energy optimized data gathering (CWMEODG) is introduced. The main aids of the suggested CWMEODG technique are summarized as follows;

- To improve the data gathering with minimum energy consumption, CWMEODG technique is developed. For each sensor node in the population, the remaining energy is calculated. In fitness calculation, the residual strength of the node is related with the threshold value. If the energy of the node is more preponderant than the threshold value, then the node is designated as a current best.

- The energy of the current best is compared with the other sensor nodes to find the global optimum through the fitness measure. The global optimum is determined by applying the chaotic tent map mathematical model to the algorithm.

- To improve the delivery of data packet, minimize the loss rate and delay, the source node propels the data packet towards the higher energy sensor node to the sink node. 
- The rest of the article is arranged into five different sections. Section 2 outlines the allied works. In section 3, the proposed CWMEODG procedure is explained with a neat diagram. In section 4, simulation setting is exhibited with various parameters. Simulation outcomes are deliberated in section 5. The deduction of paper is presented in section 6 .

\section{RELATED WORK}

An artificial bee colony (ABC) optimized particle swarm optimization procedure (ABC-PSO) was introduced in [11] for improving the lifespan of network by consuming the minimum energy. Though the algorithm improves the robustness and the reliability, energy utilization rates were not minimized at the required level. Ant Colony Optimization Algorithm was designed in [12] for dipping the node energy and enhancing the network lifespan. The optimization algorithm did not effectively provide the global optimum solution.

In [13], Vivacity Supervision and Cross-layer Optimization Method was introduced intended at reducing the time-average rate of energy depletion. The algorithm failed to perform the delay reduction in the data gathering practice. The particle swarm optimization-based clustering algorithm was developed in [14] for performing data collection with lesser consumption of energy and transmission delay using the mobile sink. The algorithm failed to minimize the packet loss in data collection.

An adaptive anchor selection algorithm was introduced in [15] using mobile collector, to amass the data from the selected sensors and maintain the consumption of energy. The algorithm failed to select optimal energy efficient sensor nodes. A Pareto-Optimal Clustering Method was introduced in [16] for data aggregation and improving the network lifetime by achieving better energy savings. But the performance of delay while performing the data gathering was not minimized.

In [17], delay-efficient traffic adaptive (DETA) method was introduced aimed at collecting the data from sensor nodes using lesser consumption of energy. Though the method considerably reduces the data collection delay, the data packet delivery proportion was not enriched. A unicast tree-based data gathering protocol (UTDG) was designed in [18] to improve the message distribution rates with minimum communication overhead as well as delay. But the data loss rate was not minimized during the data collection.

An ant colony optimization (ACO) procedure was designed in [19] using a mobile sink for gathering the data and increasing the network lifespan. The algorithm failed to transmit the data packet with minimum delay. An on-demand mobile sink traversal (ODMST) procedure was designed [20] to aggregate the information from $\mathrm{CHs}$ through the mobile sink. The convergence of procedures in large scale of WSN was not attained in an effective manner. A novel bacteria foraging optimization (BFO) algorithm was introduced in [21] for energy efficient communication in WSN. The algorithm maintains good stability between the computational and communication demands of a sensor node. However, the cost of computational complexity was not sufficient. A novel approach of ant colony optimization (ACO) algorithm was implemented in [22] for information transmission in the WSN. In this method optimal routing is based on the both the node mobility and the energy of the node. The introduced method obtain improvement in the energy consumption of the nodes per transmission. However, the performance of delay during information transmission was not considered.

A high-level adaptive power management circuit (PMC) was presented in [23] to achieve energy efficient data transmission. However, the minimization of end-to-end delay was not sufficient. A novel routing protocol for WSN's was designed in [24]. In the introduced protocol, transmitting data process is done in a hierarchal way with improved QoS in the network. However, the performance of energy minimization was not enough. An energy-efficient handover mechanism was introduced in [25] for achieving higher packet delivery ratio during data transmission in WSN. But the packet loss rate was not considered. The issues identified from the above-said reviews are overcome by presenting a new procedure called CWMEODG technique. The procedure of the CWMEODG technique is explained with the neat diagram in the next section.

\section{RESEARCH METHODOLOGY}

In WSN, several sensor nodes have the capability to sense and forwards the information to sink node. The sink node turns as a data collector to assemble the facts from all sensor nodes for future use. Throughout the data gathering process, the energy resource is essential to upsurge the lifespan of network. The sink node and sensor nodes balance the energy in the data gathering process. Based on this objective, chaotic whale metaheuristic energy optimized data gathering (CWMEODG) is introduced. The following system model is used for organizing the proposed CWMEODG technique. 


\subsection{Organization model}

Consider the wireless sensor network (WSN) arranged in the graph ' $G=(V, E)$ ' where ' $V$ ' represents a vertices i.e. number of sensor nodes denoted as ' $V=S n_{1}, S n_{2}, S n_{3} \ldots, S_{n}$ ' and a set ' $E$ ' denotes an edges i.e. connection between the nodes in a sensing zone. The nodes are organized in the square area for monitoring and collecting the data packets $d p_{1}, d p_{2}, d p_{3} \ldots d p_{n}$ and transmitting to the sink node $(S N)$ through the neighboring nodes $N N_{1}, N N_{2}, N N_{3}, \ldots . N N_{n}$. Based on the above-said system model, the proposed CWMEODG technique is designed.

\subsection{Chaotic whale metaheuristic energy optimized data gathering in WSN}

The CWMEODG technique is employed to perform the data collection in WSN with minimum energy consumption. The conventional whale optimization algorithm is still not efficient to perform the better solution. In order to attain the good convergence rate and obtain the globally optimal solution, chaotic whale metaheuristic optimization is employed by tuning the certain parameters used in the algorithm. The chaotic whale optimization algorithm is the metaheuristic which provides better solutions with minimal computational effort. The CWMEODG method expends the energy as major resource to upsurge network lifespan in data gathering process. The architecture diagram of CWMEODG technique is shown in Figure 2.

Initially, the dedicated quantity of sensor nodes is positioned in network for collecting data packets from the environmental conditions. After accumulating the data packets, the sensor nodes energy is computed to find optimal node using chaotic whale metaheuristic optimization technique. After finding the energy efficient nodes, the collected data packets are sent towards sink to enrich the network lifespan. The detailed process of CWMEODG technique is described in the following section.

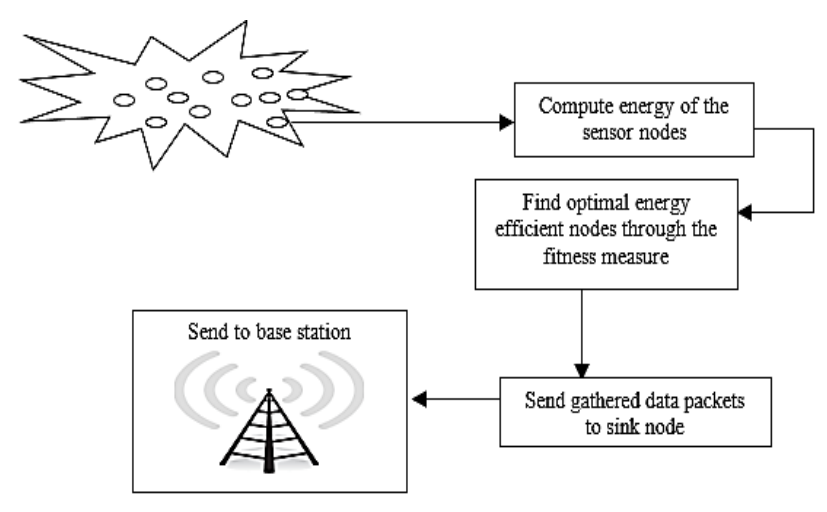

Figure 2. Architecture diagram of proposed CWMEODG technique

The populations of the sensor nodes $S n_{1}, S n_{2}, S n_{3} \ldots, S_{n}$ are initialized in the WSN for collecting the data packets $d p_{1}, d p_{2}, d p_{3} \ldots d p_{n}$ from the environment. At first, all the nodes in network have equivalent energy level. Sensor node energy is processed by the product of power and time. The energy is mathematically computed as follows,

$$
e=p * t
$$

From (1), $e$ represents the energy of the nodes, $p$ denotes a power in watts, $t$ denotes a time in seconds (Sec). The energy of every single node is quantified as unit of joule ( $\mathrm{J})$. After the data collection, the energy of each sensor node gets degraded and the remaining (i.e. residual) energy of the nodes are computed as follows,

$$
E_{r}=E_{T}-E_{c}
$$

In (2), $E_{r}$ represents the residual energy, $E_{T}$ denotes a total energy, $E_{c}$ signifies the consumed energy of sensor nodes. The nodes residual energy is used for identifying sensor nodes which utilize the more energy in the data collection. Therefore, an efficient optimization technique is used to select energy efficient nodes for data gathering in WSN. The CWMEODG technique computes the fitness of each sensor node for selecting the optimal to perform data gathering in WSN. The fitness is computed as follows,

$$
\text { Fitness }=E_{r} \geq \delta_{E}
$$


From (3), $E_{r}$ denotes a residual energy, $\delta_{E}$ represents the threshold for the residual energy. Based on the fitness measure, the current best optimal energy efficient sensor node is selected. In order to identify the global best optimal sensor nodes, three phases are carried out such as encircling prey, exploitation phase, and exploration phase. Followed by, global optimal sensor nodes are selected for data gathering in WSN.

\subsubsection{Encircling prey}

Encircling prey is the first level of the chaotic whale metaheuristic optimization technique. In this phase, the whale (i.e. sensor node) discovers the location of the prey and encircles them since the location is not known previously in the search space. Due to this, the proposed optimization algorithm considers that the current best sensor node is close to an optimal. The current best optimal sensor node is selected based on fitness computation. After finding the current best sensor node, the position of the whale is updated towards the search agent for comparing with other solution (i.e. whale) to find the energy optimized sensor node. The position update process is expressed as follows,

$$
\begin{aligned}
& x_{s_{n}}(i+1)=x_{L}(i)-B \cdot D_{t} \\
& D_{t}=\left|R \cdot x_{L}(i)-x_{s_{n}}(i)\right|
\end{aligned}
$$

In (4), $x_{s_{n}}(i+1)$ denotes an updated location of the sensor nodes, $x_{L}(i)$ represents the position vector of the prey. $B$ is a coefficient vector, $D_{t}$ represents the distance between the position vector of the prey $x_{L}(i)$ and the position vector of the whale $x_{s_{n}}(i)$. In (5), $R$ represents the coefficient vector. As mentioned above, $B$ and $R$ parameters used for shrinking the encircling mechanism. On contrary to a conventional whale optimization algorithm, the proposed whale optimization defines the two parameters $B$ and $R$ with the chaotic map value. These parameters are defined as follows,

$$
\begin{aligned}
& B=\gamma\left(2 * C_{m}-1\right) \\
& R=2 * C_{m}
\end{aligned}
$$

From (6), $\gamma$ denotes component which is decremented from 2 to 0 throughout the sequence of repetitions. $C_{m}$ denotes an assessment obtained from the chaotic map. The proposed optimization technique uses the tent chaotic map for identifying the global optimum solution. The tent is a shape of the graph which is shown in Figure 3. Figure 3 illustrates a chaotic tent map. The map function is expressed as follows,

$$
V_{n+1}=\left\{\begin{array}{lll}
\omega * v_{n} & \text { for } & v_{n}<0.5 \\
\omega *\left(1-v_{n}\right) & \text { for } & v_{n} \geq 0.5
\end{array}\right.
$$

From (8), $V_{n+1}$ is the real valued function of the chaotic tent map in the range from 0 to 1 in the unit interval. Hence the chaotic tent map is called as a discrete-time dynamical system. $v_{n}$ is parameter ranges from 0 to 1 and choosing the value of parameter $\omega=2$.

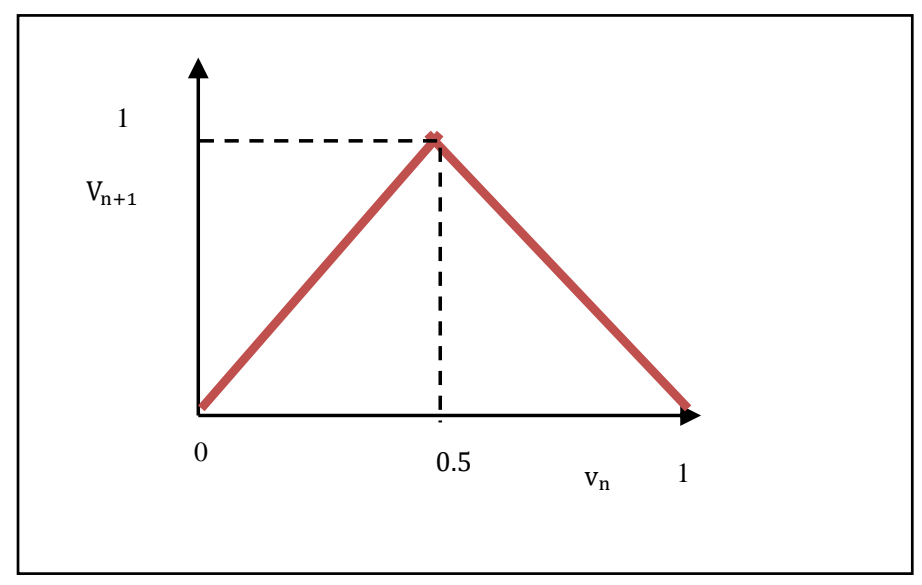

Figure 3. Chaotic tent map 


\subsubsection{Exploitation phase}

The exploitation phase is the second level of the optimization technique. This segment is also known as a Bubble-net attacking technique. In the exploitation phase, the distance between the whale and location of the prey is computed. In this phase, a helix equation is formulated with the location of the prey and the whale's location to follow the helix-shaped motion of humpback whales. The updating results are expressed using the chaotic tent map as follows,

$$
\begin{aligned}
& x_{s_{n}}(i+1)=D_{t}{ }^{\prime} * e^{\alpha C_{m}} \cos \left(2 \pi C_{m}\right)+x_{s_{n}}{ }^{\prime}(i) \\
& D_{t}{ }^{\prime}=\left|x_{L}(i)^{\prime}-x_{s_{n}}(i)\right|
\end{aligned}
$$

In (10), $x_{s_{n}}(i+1)$ represents the update position of the sensor nodes, $D_{t}{ }^{\prime}$ denotes an updated distance between the ' $i$ 'th whale position $x_{S_{n}}(i)$ to the prey position $x_{L}(i)$ ', $\alpha$ denotes a constant for outlining the logarithmic curve structure. Exponential function ' $e$ ' is the base of natural logarithms. $C_{m}$ represents the value of the chaotic tent map $(0,1)$.

The humpback whales enclose the prey in a dwindling circle and also a coiled structured pathway concurrently. In order to perform these concurrent performances, consider that there is a possibility of 0.5 to elect either dwindling circle or helix-shaped model. The precise model is expressed as follows,

$$
x_{s_{n}}(i+1)= \begin{cases}x_{s_{n}}{ }^{\prime}(i)-B \cdot D_{t} & ; \quad P<0.5 \\ D_{t}{ }^{\prime} * e^{\alpha C_{m}} \cos \left(2 \pi C_{m}\right)+x_{s_{n}}{ }^{\prime}(i) & ; \quad P \geq 0.5\end{cases}
$$

In $(11), x_{s_{n}}(i+1)$ represents the updated position of the sensor nodes, ' $P$ ' is a probability ranges from $[0,1]$.

\subsubsection{Exploration phase}

The final phase of the proposed chaotic whale metaheuristic optimization technique is the exploration phase i.e. searches for prey. The global optimum solution is obtained by updating the whale's position with a randomly chosen whale rather than the current best whale. This mechanism uses the $|R|>1$ which highlights the exploration and permits the proposed chaotic whale metaheuristic optimization algorithm that performs a global search. The updating behavior is expressed as follows:

$$
\begin{aligned}
& D_{t}=\left|R \cdot x_{\text {rand }}(i)-x_{s_{n}}(i)\right| \\
& x_{s_{n}}(i+1)=x_{\text {rand }}(i)-B \cdot D_{t}
\end{aligned}
$$

From (13), $x_{\text {rand }}(i)$ denotes an arbitrary location vector of a haphazard whale. Finally, the chaotic whale metaheuristic optimization algorithm is stopped by satisfying the termination criterion. By this way, energy efficient sensor nodes are selected from the population. After that, the lesser energy sensor nodes are employed to direct the collected data packets towards nearest higher energy sensor nodes for transferring the packets to the sink node. The Euclidean distance measure is used for finding neighboring higher energy nodes in the search space. The Euclidean distance is computed as follows,

$$
d_{E}=\sqrt{\sum\left(E_{l}\left(s_{n}\right)-E_{h}\left(s_{n}\right)\right)^{2}}
$$

From (14), $d_{E}$ represents the Euclidean distance, $E_{l}\left(s_{n}\right)$ denotes lesser energy sensor nodes and $E_{h}\left(s_{n}\right)$ represents the higher energy sensor nodes. Lesser energy sensor nodes find the neighboring higher energy sensor nodes to transmit the packets towards sink. After that, the source node sends accumulated packets to the sink through the adjacent higher strength sensor nodes.

Figure 4 illustrates a data gathering through the optimal energy efficient sensor nodes. In Figure 4, the source node (S) propels the data packet to nearest high energy sensor nodes which is represented in green color. The higher energy sensor nodes direct the data packets towards sink node represented in red color. The sink node collects data packets from the higher energy nodes that ensues in refining the lifetime of network.

The algorithmic explanation of CWMEODG technique is presented as follows, Input: Number of sensor nodes (i.e. whale) $S n_{1}, S n_{2}, S n_{3} \ldots, S_{n}$, Number of data packets $d p_{1}, d p_{2}, d p_{3} \ldots d p_{n}$ Output: Improved energy efficient data gathering 


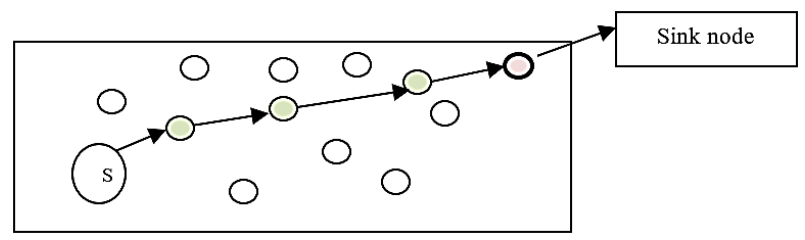

Figure 4. Data gathering in WSN

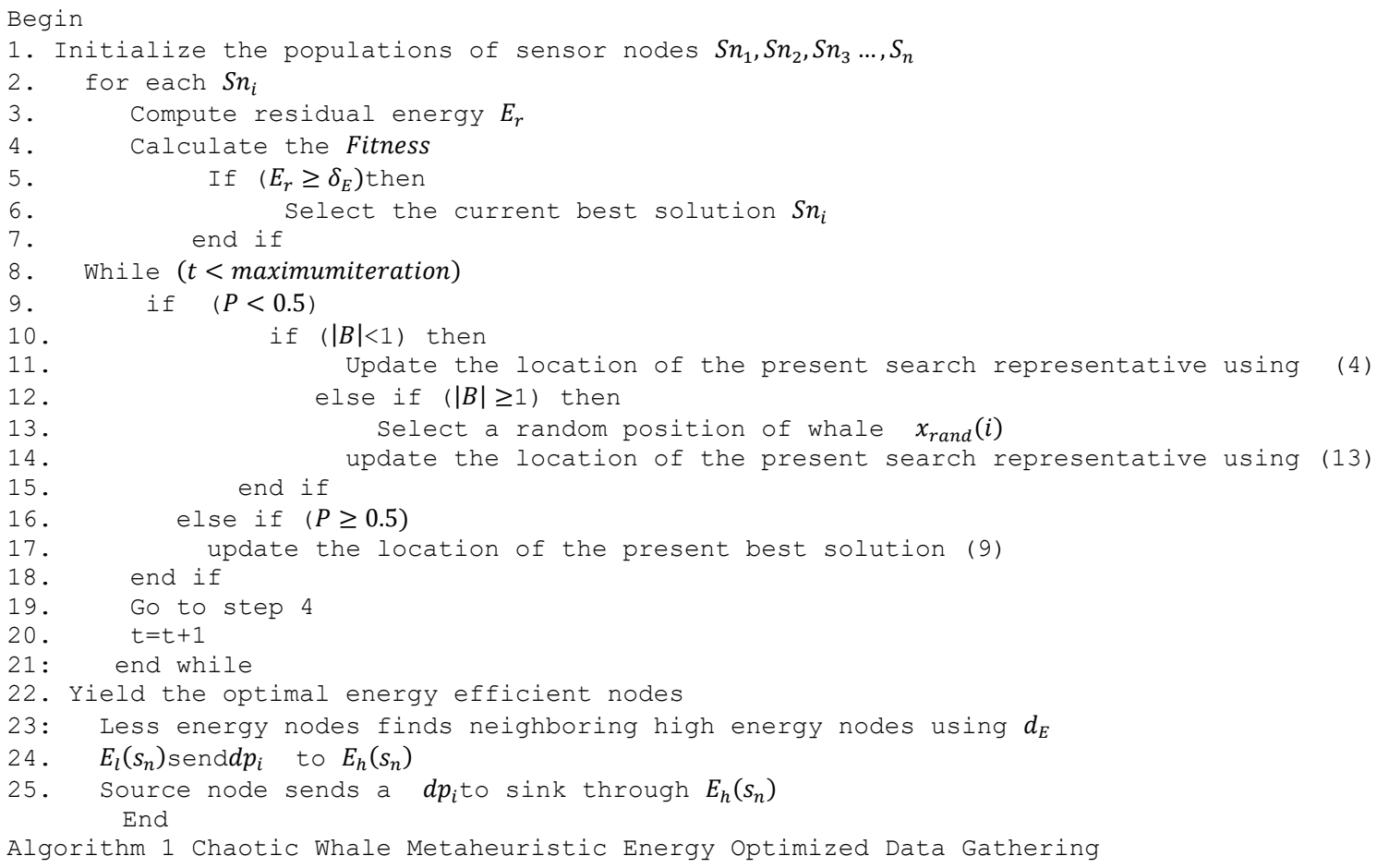

Algorithm 1 describes the chaotic whale metaheuristic energy optimized data gathering with minimum delay and loss. The populations of the sensor nodes are initialized arbitrarily. For each node, residual energy is assessed. Reliant on the energy, the fitness is computed for each sensor nodes. The residual energy is compared by means of the threshold value for identifying the current best search agent (i.e. sensor nodes). After finding the current best agent, the proposed CWMEODG technique performs three different processes by tuning the certain parameters using chaotic tent map values. After that, the fitness is computed to find the global energy efficient sensor nodes. Predicated on the tent map, the fast convergence of the algorithm is attained and selects the energy efficient sensor nodes among the dedicated quantity of nodes deployed in the network. After finding the energy efficient nodes, the lesser energy sensor nodes transfer the composed data to the nearby optimal energy efficient node through the Euclidean distance measure. The source node then directs the collected packets to the sink over the energy efficient sensor nodes with minimum delay. This process upsurges the packet delivery proportion and network lifetime. The above-explained algorithmic processes are executed in the simulation to show the performance of the suggested CWMEODG technique than the existing optimization technique. The simulation results are discussed in next section.

\section{SIMULATION SETTINGS}

An efficient CWMEODG technique and existing methods PSOBS [1] and ACOPSO [2] are implemented in NS2.34 network simulator. Totally 500 sensor nodes are distributed in a square region of $A^{2}(1500 \mathrm{~m} * 1500 \mathrm{~m})$ for data gathering in WSN. The mobility model in the simulation is used as Random Waypoint. The number of data packets used for the simulation purposes are varied from 25 to 250. The sensor nodes speed is set as $0-20 \mathrm{~m} / \mathrm{sec}$ and the simulation time is $300 \mathrm{sec}$. The dynamic source routing (DSR) protocol is applied for carrying out energy effective data gathering in WSN. The various simulation factors and their values are listed in Table 1. 
The model is performed with reverence to quantity of sensor nodes and data packets. The various performance metrics such as energy utilization, packet delivery proportion, loss rate of data packet and delay are computed using the above said simulation settings. The results of different parameters are discussed in next section.

Table 1. Simulation factors

\begin{tabular}{ll}
\hline Simulation Factors & Values \\
\hline Simulator & NS2.34 \\
Network Range & $1500 \mathrm{~m} * 1500 \mathrm{~m}$ \\
Quantity of sensor nodes & $50,100,150,200,250,300,350,400,450,500$ \\
Mobility model & Random Way point model \\
Quantity of Data packets & $25,50,75,100,125,150,175,200,225,250$ \\
Sensor nodes speed & $0-20 \mathrm{~m} / \mathrm{s}$ \\
Simulation Time & $300 \mathrm{sec}$ \\
Number of Tracks & 10 \\
Protocol & DSR \\
\hline
\end{tabular}

\section{RESULTS AND DISCUSSIONS}

The simulation outcomes of projected CWMEODG technique and existing optimization techniques namely PSOBS [1] and ACOPSO [2] are discussed with various parameters such as consumption of energy, data packet delivery proportion, data packet loss rate and delay. Performance of CWMEODG technique is evaluated with the existing methods using table values and graphical results. For each section, the sample mathematical calculation is provided for showing the performance results of the proposed CWMEODG technique and existing methods.

\subsection{Simulation results of energy utilization}

Consumption of energy is calculated as an amount of vitality spent by sensor nodes for sensing and gathering the data from the environmental conditions. The mathematical formula for calculating the energy consumption is expressed as follows,

$$
\text { Energy consumption }=\text { Number of sensor nodes } * E_{C}(\text { single sensor node })
$$

From (15), $E_{C}$ denotes energy consumption of sensor node. The consumption of energy is restrained in terms of joule (J). Sample mathematical calculation for energy consumption:

- Proposed CWMEODG technique: Total quantity of sensor nodes are taken as 50, energy consumption for single sensor node is $0.54 \mathrm{~J}$ oule, then the total energy consumption is computed as,

$$
\text { Energyconsumption }=50 * 0.54 \mathrm{Joule}=27 \mathrm{Joule}
$$

- Existing PSOBS: Total number of sensor nodes are taken as 50, energy consumption for single sensor node is $0.65 \mathrm{~J}$ oule, then the total energy consumption is computed as,

$$
\text { Energyconsumption }=50 * 0.65 \mathrm{Joule}=32.5 \mathrm{Joule} \approx 33 \mathrm{Joule}
$$

- Existing ACOPSO: Total number of sensor nodes are taken as 50, energy consumption for single sensor node is $0.8 \mathrm{~J}$ oule, then the total energy consumption is computed as,

$$
\text { Energy consumption }=50 * 0.8 \mathrm{Joule}=40 \mathrm{Joule}
$$

The simulation outcomes of the energy utilization of the sensor nodes via three different optimization techniques namely CWMEODG, PSOBS [1] and ACOPSO [2] are described in Table 2. For the simulation purposes, the definite quantity of sensor nodes is taken from 50 to 500 . The various number of sensor nodes are considered and it reported for each instance. At first 50 number of sensor nodes are considered, 27J energy consumption is attained in the proposed CWMEODG, existing PSOBS [1] and ACOPSO [2] attains 33J and 40J respectively. In this calculation, the nodes energy utilization is computed after sensing and gathering the data. The results illustrate that the proposed CWMEODG technique consumes lesser energy than the conventional optimization technique. 
Totally ten various runs are performed through different quantity of sensor nodes. The outcomes of the CWMEODG scheme is compared by means of two present techniques PSOBS [1] and ACOPSO [2]. The comparison results show that the CWMEODG technique minimizes consumption of energy by $12 \%$ and $19 \%$ when related to existing optimization techniques.

Table 2. Tabularization for energy utilization

\begin{tabular}{cccc}
\hline Quantity of Sensor nodes & \multicolumn{3}{c}{ Energy consumption (Joule) } \\
& CWMEODG & PSOBS & ACOPSO \\
\hline 50 & 27 & 33 & 40 \\
100 & 35 & 39 & 45 \\
150 & 39 & 47 & 51 \\
200 & 42 & 50 & 54 \\
250 & 46 & 53 & 58 \\
300 & 48 & 56 & 60 \\
350 & 55 & 59 & 63 \\
400 & 57 & 61 & 64 \\
450 & 61 & 65 & 69 \\
500 & 63 & 68 & 73 \\
\hline
\end{tabular}

\subsection{Simulation results of data packet delivery ratio}

Data packet delivery ratio is measured as the proportion of the quantity of packet correctly received at the sink node to the total quantity of packets propelled from the source node. The data packet delivery ratio is precisely computed as follows,

$$
D P D R=\frac{\text { No.ofDP }}{N o . o f D P_{S}} * 100
$$

From (16), $D P D R$ denotes data packet delivery ratio, $D P_{r}$ denotes a data packet correctly received, $D P_{S}$ represents a data packet propelled from source node. The packet delivery ratio is measured in percentage $(\%)$. Sample mathematical calculation for data packet delivery ratio:

- Proposed CWMEODG technique: Definite quantity of packets received at sink node is 21 and the quantity of packets sent is 25 . Then the data packet delivery ratio is computed as,

$$
D P D R=\frac{21}{25} * 100=84 \%
$$

- Existing PSOBS: Definite quantity of packets received at sink node is 20 and the quantity of packets sent is 25 . Then the data packet delivery ratio is computed as,

$$
D P D R=\frac{20}{25} * 100=80 \%
$$

- Existing ACOPSO: Definite quantity of packets received at sink node is 19 and the quantity of packets sent is 25 . Then the data packet delivery ratio is computed as,

$$
D P D R=\frac{19}{25} * 100=76 \% \text {. }
$$

Table 3 describes the various simulation results of data packet delivery ratio with deference to a definite quantity of sensor nodes in WSN. The model outcomes clearly show that the CWMEODG method increases the data packet delivery ratio when analyzed to other techniques PSOBS [1] and ACOPSO [2]. In Table 3, let us consider the number of data packets are 25, 21 packets correctly accepted at sink node using CWMEODG technique and their percentage is $84 \%$. The definite quantity of data packets acceptable at the sink node using PSOBS [1] and ACOPSO [2] are 20 and 19 respectively. The equivalent percentage value of PSOBS [1] and ACOPSO [2] are $80 \%$ and $76 \%$ respectively.

After performing the ten occurrences with a different count of packets, the proposed data packet delivery results are compared using the existing results. Then the average value is taken for the comparison results. The average results prove that the proposed CWMEODG technique considerably amends the data packet delivery ratio by $6 \%$ compared to the PSOBS [1]. Similarly, the proposed CWMEODG technique also increases the data packet delivery ratio by $12 \%$ compared with the other existing technique ACOPSO [2]. 
Table 3. Tabularization for data packet delivery ratio

\begin{tabular}{cccc}
\hline Quantity of Data Packets & \multicolumn{3}{c}{ Data packet delivery ratio (\%) } \\
& CWMEODG & PSOBS & ACOPSO \\
\hline 25 & 84 & 80 & 76 \\
50 & 90 & 86 & 82 \\
75 & 89 & 84 & 77 \\
100 & 88 & 83 & 79 \\
125 & 85 & 81 & 77 \\
150 & 83 & 79 & 75 \\
175 & 89 & 81 & 77 \\
200 & 90 & 86 & 81 \\
225 & 88 & 84 & 82 \\
250 & 89 & 82 & 78 \\
\hline
\end{tabular}

\subsection{Simulation results of data packet loss rate}

Data packet loss rate is assessed as the proportion of definite quantity of packets lost at sink node to the total quantity of packets propelled from the source node. The data packet loss rate is computed using the mathematical equation,

$$
D P L R=\frac{\text { No.ofDP }}{N o . o f D P_{S}} * 100
$$

From (17), DPLR denotes data packet loss rate, $D P_{L}$ denotes a data packet loss, $D P_{S}$ represents data packet sent from source node. The data packet loss rate is assessed in percentage (\%). Sample mathematical calculation for data packet loss rate:

- Proposed CWMEODG technique: Definite quantity of data packets lost at the sink node is 4 and quantity of data packets transmitted is 25 . The packet loss rate is calculated as follows,

$$
D P L R=\frac{4}{25} * 100=16 \%
$$

- Existing PSOBS: Definite quantity of data packets lost at the sink node is 5 and quantity of data packets transmitted is 25 . The packet loss rate is calculated as follows,

$$
D P L R=\frac{5}{25} * 100=20 \%
$$

- Existing ACOPSO: Definite quantity of data packets lost at the sink node is 6 and quantity of packets transmitted is 25 . The packet loss rate is calculated as follows,

$$
D P L R=\frac{6}{25} * 100=24 \% \text {. }
$$

Table 4 describes a data packet loss rate versus a quantity of data packets. The loss rate is measured at the sink node which results in improving the data gathering efficiency when compared to conventional optimization methods. The sink node acts like a data collector and gathers the data from the higher energy sensor nodes.

Table 4. Tabularization for data packet loss rate

\begin{tabular}{cccc}
\hline Quantity of Data Packets & Data packet loss rate (\%) & \\
& CWMEODG & PSOBS & ACOPSO \\
\hline 25 & 16 & 20 & 24 \\
50 & 10 & 14 & 18 \\
75 & 11 & 16 & 23 \\
100 & 12 & 17 & 21 \\
125 & 15 & 19 & 23 \\
150 & 17 & 21 & 25 \\
175 & 11 & 19 & 23 \\
200 & 10 & 14 & 19 \\
225 & 12 & 16 & 18 \\
250 & 11 & 18 & 22 \\
\hline
\end{tabular}


In the simulation results, while considering 25 data packets, 4 data packets get lost using proposed CWMEODG technique owing to random transfer of sensor nodes in the network. As a consequence, $12 \%$ of data packet loss is attained. Whereas 5 and 6 packets are lost using PSOBS [1] and ACOPSO [2] technique and their loss percentages are $20 \%$ and $24 \%$ respectively. Therefore, the energy efficient data gathering is attained using CWMEODG technique while minimizing the data packet loss. The loss rate of CWMEODG technique is considerably minimized by $28 \%$ and $42 \%$ when compared to existing PSOBS [1] and ACOPSO [2] technique.

\subsection{Simulation results of delay}

Data gathering delay is outlined as the variance between the time for the packet transmitted from the source node and the period of time at which the definite quantity of data packets attained at the sink node. The delay is computed using the following equation,

$$
D=T_{A}-T_{S}
$$

in (18), $D$ represents the delay, $T_{R}$ denotes an arrival time, $T_{S}$ represents the sending time. The delay is measured in milliseconds (ms). Sample mathematical calculation for delay:

- Proposed CWMEODG technique: Let us consider the number of data packets are 25, packet arrival time is $11 \mathrm{~ms}$ and the initial time for the data packets starts to send from the source node is considered as $0 \mathrm{~ms}$. Then delay at the sink node is computed as,

$$
D=11 m s-0 m s=11 m s
$$

- Existing PSOBS: Let us consider the number of data packets are 25, packet arrival time is $13 \mathrm{~ms}$ and the initial time for the data packets starts to send from the source node is considered as 0ms. Then delay at the sink node is computed as,

$$
D=13 m s-0 m s=13 m s
$$

- Existing ACOPSO: Let us consider the number of data packets are 25, packet arrival time is $15 \mathrm{~ms}$ and the initial time for the data packets starts to send from the source node is considered as0ms. Then the delay is calculated as follows,

$$
D=15 m s-0 m s=15 m s
$$

Table 5 describes simulation results of delay with point to a definite quantity of packets conveyed from source node. The delay is mathematically deliberated based on the difference between the sending and arrival time of data packets. The above table shows that the ten various results of delay using three different optimization techniques namely CWMEODG, PSOBS [1], ACOPSO [2]. Among the three different techniques, the performance of the delay is significantly minimized using CWMEODG method.

Let us consider the 25 packets dispatched from source node, the simulation result of proposed CWMEODG technique obtains a delay of $11 \mathrm{~ms}$. The delay of other existing PSOBS [1], ACOPSO [2] are $13 \mathrm{~ms}$ and $15 \mathrm{~ms}$ respectively. Compared to existing methods, the proposed CWMEODG technique effectively improves the energy efficient data gathering with minimum delay. As a result, the delay of ten different results of CWMEODG technique is minimized by $11 \%$ and $22 \%$ when compared to existing PSOBS [1], ACOPSO [2] respectively.The above discussion effectively improves the energy efficient data gathering with minimal energy consumption, delay, loss and higher packet delivery ratio.

Table 5. Tabularization for delay

\begin{tabular}{cccc}
\multicolumn{4}{c}{ Table 5. Tabularization for delay } \\
\hline \multirow{2}{*}{ Quantity of Data Packets } & \multicolumn{3}{c}{ Delay (ms) } \\
& CWMEODG & PSOBS & ACOPSO \\
\hline 25 & 11 & 13 & 15 \\
50 & 13 & 15 & 18 \\
75 & 15 & 18 & 20 \\
100 & 17 & 19 & 22 \\
125 & 18 & 20 & 23 \\
150 & 21 & 23 & 26 \\
175 & 23 & 26 & 29 \\
200 & 24 & 27 & 30 \\
225 & 26 & 29 & 31 \\
250 & 28 & 30 & 33 \\
\hline
\end{tabular}

Int J Elec \& Comp Eng, Vol. 10, No. 4, August 2020 : 4176 - 4188 


\section{CONCLUSION}

An efficient technique called CWMEODG is developed to amend the energy proficient data amassing in sensor networks with minimal delay. The energy efficient nodes are determined through the chaotic whale metaheuristic optimization. After that, the source node transfers the packets to the sink with the aid of energy efficient sensor nodes by enhancing the network lifespan, increasing the packet delivery proportion and minimizes the data loss rate. In CWMEODG technique, the sink node gathers the data packet from the higher energy nodes instead of collecting from all the nodes in WSN. As per consequence, the delay at the sink is minimized. The simulation is carried out with different parameters such as energy consumption, data packet delivery ratio, packet loss rate and delay. The performance consequences clarifies that the CWMEODG technique improves the energy efficient data gathering with high delivery ratio and minimal delay as well as packet loss when examined to the former methods.

\section{REFERENCES}

[1] Shamineh Tabibi and Ali Ghaffari, "Energy-Efficient Routing Mechanism for Mobile Sink in Wireless Sensor Networks Using Particle Swarm Optimization Algorithm," Wireless Personal Communications, Springer, vol. 104, no. 1, pp. 199-216, Jan. 2019.

[2] Supreet Kaur and Rajiv Mahajan, "Hybrid Meta-heuristic Optimization-Based Energy-Efficient Protocol for Wireless Sensor Network," Egyptian Informatics Journal, vol. 19, no. 3, pp. 145-150, Nov. 2018.

[3] Ngoc-Tu Nguyena, Bing-Hong Liu, Van-Trung Pham and Yi-Sheng Luo, "On Maximizing the Lifetime for Data Aggregation in Wireless Sensor Networks Using Virtual Data Aggregation Trees,” Computer Networks, vol. 105, pp. 99-110, Aug. 2016.

[4] Soo-Hoon Moona, Sunju Park and Seung-jae Han, "Energy Efficient Data Collection in Sink-Centric Wireless Sensor Networks: A Cluster-Ring Approach,” Computer Communications, vol. 101, pp. 12-25, Mar. 2017.

[5] Saud Althunibat, Amir Abu-Al-Aish, Wael F. Abu Shehab and Wael H. Alsawalmeh, "Auction-Based Data Gathering Scheme for Wireless Sensor Networks," IEEE Communications Letters, vol. 20, no. 6, pp. 1223-1226, Jun. 2016.

[6] Xiangping Gu, Xiaofeng Zhou, Baohua Yuan and Yanjing Sun, "A Bayesian Compressive Data Gathering Scheme in Wireless Sensor Networks With One Mobile Sink," IEEE Access, vol. 6, pp. 47897-47910, 2018.

[7] Chul-Ho Lee, Jaewook Kwak and Do Young Eun, "Towards Distributed Optimal Movement Strategy for Data Gathering in Wireless Sensor Networks," IEEE Transactions on Parallel and Distributed Systems, vol. 27, no. 2, pp. 574-584, Feb. 2016.

[8] Charalampos Konstantopoulos, Nikolaos Vathis, Grammati Pantziou and Damianos Gavalas, "Employing Mobile Elements for Delay-Constrained Data Gathering in WSNs," Computer Networks, vol. 135, pp. 108-131, Apr. 2018.

[9] Prabhudutta Mohanty and Manas Ranjan Kabat, "Energy Efficient Structure-Free Data Aggregation and Delivery in WSN," Egyptian Informatics Journal, vol. 17, no. 3, pp. 273-284, Nov. 2016.

[10] Cuicui Lv, Qiang Wang, Wenjie Yan and Jia Li, "A Sparsity Feedback-Based Data Gathering Algorithm for Wireless Sensor Networks,” Computer Networks, vol. 141, pp. 145-156, 4 Aug. 2018.

[11] Yinggao Yue, Li Cao, Bo Hang and Zhongqiang Luo, "A Swarm Intelligence Algorithm for Routing Recovery Strategy in Wireless Sensor Networks with Mobile Sink," IEEE Access, vol. 6, pp. 67434-67445, 2018.

[12] SnehaSuhas More and Mininath K. Nighot, "Optimization of Wireless Sensor Networks Using Artificial Intelligence and Ant Colony Optimization to Increase Network Lifetime and to Minimize Energy of Network," 2017 International Conference on Computing, Communication, Control and Automation, pp. 1-6, 2017.

[13] Weiqiang Xu, Yushu Zhang, Qingjiang Shi and Xiaodong Wang, "Energy Management and Cross Layer Optimization for Wireless Sensor Network Powered by Heterogeneous Energy Sources," IEEE Transactions on Wireless Communications, vol. 14, no. 5, pp. 2814-2826, May 2015.

[14] Jin Wang, Yiquan Cao, Bin Li, Hye-jin Kim and Sungyoung Lee, "Particle Swarm Optimization Based Clustering Algorithm with Mobile Sink for WSNs," Future Generation Computer Systems, vol. 76, pp. 452-457, Nov. 2017.

[15] Cong Wang, Songtao Guo and Yuanyuan Yang, "An Optimization Framework for Mobile Data Collection in Energy-Harvesting Wireless Sensor Networks," in IEEE Transactions on Mobile Computing, vol. 15, no. 12, pp. 2969-2986, Dec. 2016.

[16] Puneet Azad and Vidushi Sharma, "Pareto-Optimal Clustering Scheme Using Data Aggregation for Wireless Sensor Networks," International Journal of Electronics, vol. 102, no. 7, pp. 1165-1176, 2015.

[17] Byungseok Kang, Phuc Nguyen and Hyunseung Choo, "Delay-Efficient Energy-Minimized Data Collection with Dynamic Traffic in WSNs," in IEEE Sensors Journal, vol. 18, no. 7, pp. 3028-3038, Apr. 2018

[18] Mottaghinia Zeynab and Ghaffari Ali, "A Unicast Tree-Based Data Gathering Protocol for Delay Tolerant Mobile Sensor Networks," Journal of Information Systems and Telecommunication, vol. 4, no. 1, pp. 59-69, 2016.

[19] Jin Wang, Jiayi Cao, R. Simon Sherratt and Jong Hyuk Park, "An Improved Ant Colony Optimization-Based Approach with Mobile Sink for Wireless Sensor Networks," The Journal of Supercomputing, Springer, vol. 74, no. 12, pp. 6633-6645, Dec. 2018.

[20] Santosh Soni and Manish Shrivastava, "Novel Learning Algorithms for Efficient Mobile Sink Data Collection Using Reinforcement Learning in Wireless Sensor Network," Wireless Communications and Mobile Computing, Hindawi, vol. 2018, pp. 1-13, Aug. 2018. 
[21] Hemavathi. P and Nandakumar A. N., "Novel Bacteria Foraging Optimization for Energy-efficient Communication in Wireless Sensor Network," International Journal of Electrical and Computer Engineering (IJECE), vol. 8, no. 6, pp. 4755-4762, Dec. 2018.

[22] Afsah Sharmin, F. Anwar and S. M. A. Motakabber, "A Novel Bio-inspired Routing Algorithm Based on ACO for WSNs," Bulletin of Electrical Engineering and Informatics (BEEI), vol. 8, no. 2, pp. 718-728, Jun. 2019.

[23] M. S. Tamrin and M. R. Ahmad, "Simulation of Adaptive Power Management Circuit for Hybrid Energy Harvester and Real-time Sensing Application," International Journal of Power Electronics and Drive Systems (IJPEDS), vol. 11, no. 2, pp. 658-666, Jun. 2020.

[24] Salam Al-Khammasi, Dheyaa Alhelal and Nabeel Salih Ali, "Energy Efficient Cluster Based Routing Protocol for Dynamic and Static Nodes in Wireless Sensor Network," TELKOMNIKA Telecommunication, Computing, Electronics and Control, vol. 16, no. 5, pp. 1974-1981, Oct. 2018.

[25] Mahdi Zareei, Muhammed I. Alghamdi, A. K. M. Muzahidul Islam, Sabariah Baharun and Rahmat Budiarto, "Energy Efficient Handover Management in Cluster Based Wireless Sensor Network," Proceeding of the Electrical Engineering, Computer Science and Informatics, vol. 1, pp. 1-4, 2014.

\section{BIOGRAPHIES OF AUTHORS}

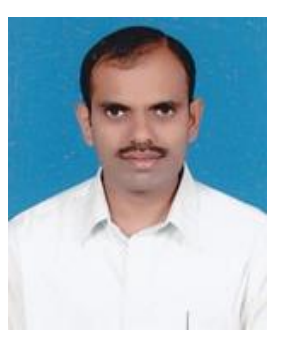

Sridhar R. is working as an Assistant Professor in Department of ISE at Global Academy of Technology, Bengaluru. He is currently pursuing the Ph.D. degree in Computer Science and Engineering affiliated to Visvesvaraya Technological University. His research interests include wireless sensor networks and Artificial intelligence.

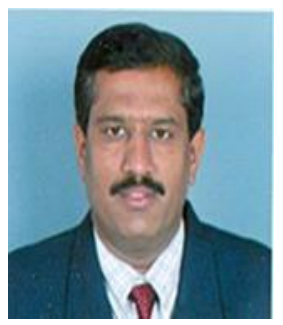

N. Guruprasad is currently working as Professor in the Department of Computer Science and Engineering, at GLOBAL ACADEMY OF TECHNOLOGY, Bengaluru. Basically a graduate, post graduate and doctorate from the field of Computer Science having 26+ years of teaching experience. Area of interest includes Programming in C \& Data Structures, Object Oriented Programming with $\mathrm{C}++$, Analysis and Design of Algorithms, Internet Technologies, UNIX and LINUX, Numerical Analysis and Operations Research. 